\title{
Iliac vein perforation caused by an ultrasound-accelerated thrombolysis catheter: An unusual complication of EKOS EndoWave
}

\author{
Hamit Serdar Başbuğ, Macit Bitargil, Hakan Göçer, Yalçın Günerhan, Kanat Özışık \\ Received: February 06, 2016 Accepted: March 11, 2016 Published online: July 21, 2016
}

\begin{abstract}
EKOS EndoWave system is an ultrasound-accelerated catheter-directed thrombolysis device designed for the treatment of deep vein thrombosis. This system acts by emitting ultrasonic waves that enhance the diffusion of the simultaneously administered thrombolytic infusion inside the clot. EKOS operates inside the thrombosed veins and is categorized as such among the group of pharmaco-mechanical thrombolysis devices. In this article, we present a case of an iliac vein perforation and extravasation of the EKOS EndoWave system that has not been encountered in the current practice thus far.
\end{abstract}

Keywords: Catheter-directed; complication; deep vein thrombosis; thrombolysis; ultrasound-accelerated.

Deep venous thrombosis (DVT) and pulmonary embolism (PE) can develop as a complication of venous thromboembolism (VTE). Venous thromboembolismassociated morbidity and mortality are frequently seen during everyday life and hospital stays. ${ }^{[1]}$ The primary therapy for the VTE is oral or parenteral anticoagulation medication provided there is no contraindication. ${ }^{[2]}$ After the initial anticoagulation medication, maintenance treatments should be administered to prevent recurrences, embolism and postthrombophlebitic syndrome (PTS). With proper treatment, the spontaneous lysis and recanalization rate of the thrombus can be as low as $10 \%$ in proximal (iliofemoral and iliocaval) DVT. ${ }^{[3]}$ Likewise, administration of systemic thrombolysis therapy can constitute a risk of major bleeding not strictly restricted to the thrombotic area. ${ }^{[4]}$ Catheter-directed ultrasound-enhanced thrombolysis devices provide a combination of the local thrombolytic infusion together with a mechanical thrombolysis action. ${ }^{[5]}$ In this article, we presented an unusual case with an iliac vein perforation and extravasation of an operating ultrasound-enhanced thrombolysis catheter following a sudden movement of thigh hyperflexion.

\section{CASE REPORT}

A 39-year-old male was admitted to the emergency department with a complaint of pain and swelling in his left leg. His symptoms had been present for the last week and had progressively gotten worse. On inspection, his left thigh circumference was remarkably larger than the right. On physical examination, his left thigh was tender to the touch, and there was a moderate pretibial edema with intact peripheral pulses. He was unable to stand and also unable to bend his left leg. Color Doppler ultrasound examination revealed a DVT starting from the popliteal vein proceeding upwards to the common iliac vein. After diagnosis as a subacute DVT, the patient decided to be treated with an ultrasound-enhanced catheterdirected thrombolysis system (EndoWave System, EKOS Corporation, Bothell, WA, USA). A written informed consent was obtained from the patient.

The patient was taken to the operating room, and he layed in a prone position. The left popliteal vein was accessed percutaneously by $7 \mathrm{~F}$ sheath via Seldinger technique under local anesthesia. An ultrasound was used to facilitate the percutaneous access. A 0.035-inch hydrophilic guide wire (Terumo Corporation, Shibuya-ku, Tokyo, Japan) was progressed to the inferior vena cava through the thrombotic segment. The infusion delivery catheter was advanced over the wire until the tip of the catheter passed through the thrombotic section. The guide wire was removed and

Department of Cardiovascular Surgery, Medical Faculty of Kafkas University, Kars, Turkey

Corresponding author: Hamit Serdar Başbuğ, MD. Kafkas Üniversitesi Tıp Fakültesi Kalp ve Damar Cerrahisi Anabilim Dalı, 36100 Kars, Turkey.

Tel: +90 474 - 2251190 e-mail: s_basbug@hotmail.com 


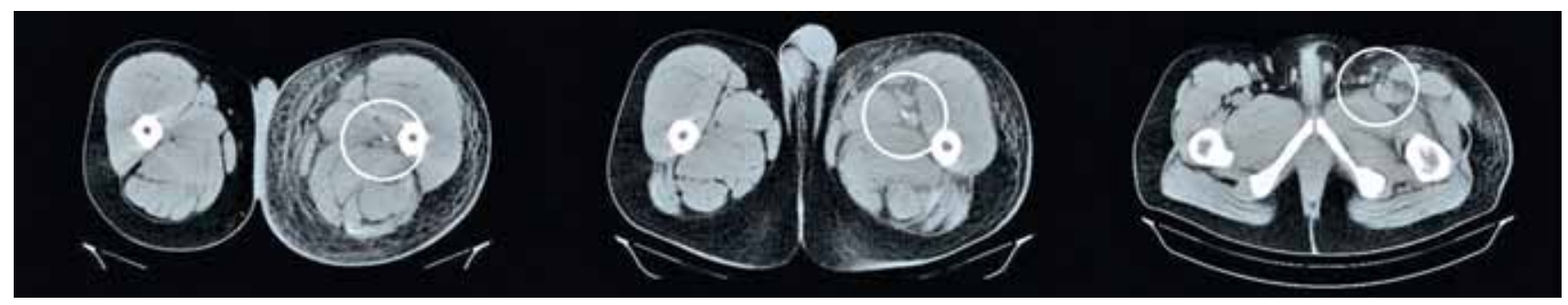

Figure 1. Computed tomography angiography scans showing the upward course of the EKOS EndoWave catheter from the popliteal vein to the common femoral vein. Note the subcutaneous changes of the left leg caused by the edema.

the microsonic ultrasound core containing multiple ultrasound transducers was inserted into the delivery catheter. The infusion of the lytic drug (Actilyze) started in the ultrasonic core. The whole process was overseen using the $\mathrm{C}$-armed fluoroscopy.

After the implantation of the ultrasound-enhanced catheter, he was taken to the in-patient ward where he spent the next 24 hours in the EKOS EndoWave System operation. However, after 10 hours of the ultrasoundenhanced catheter-directed thrombolysis process, he started to feel a burning and piercing pain in his left lower abdominal wall. On anamnesis, he described a sudden involuntary movement of hyperflexion of the left thigh while asleep during the night. On physical examination, his lower left abdomen was tender, and he was feeling increased pain during external compression. The ultrasonic catheter was immediately stopped, and a computed tomography (CT) scan was performed to visualize the course and the tip of the catheter. The course of the catheter was normal until the external iliac vein (Figure 1). However, the anteroposterior plain $\mathrm{X}$-ray and $\mathrm{CT}$ views revealed that the catheter had ripped during its route perforating the iliac vein and had advanced into the rectus abdominalis muscle (Figure 2). The transverse slices of the CT showed that the extravasation point was somewhere at the external iliac vein level (Figure 3). The EKOS EndoWave catheter system was entirely withdrawn and removed. The patient's abdominal pain instantly faded away.

The abdominal examination was repeated with frequent intervals so as not to miss an intraabdominal
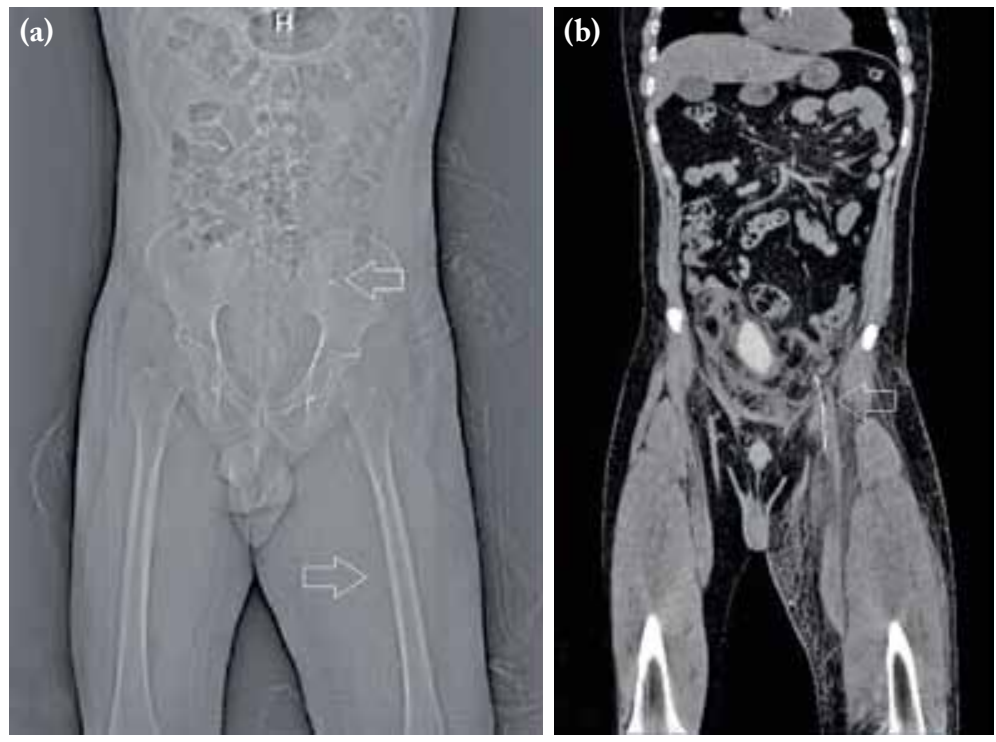

Figure 2. Plain anteroposterior roentgenogram showing the course of the EKOS EndoWave catheter. (a) Note the upper arrow that points to the extravasated tip of the catheter. (b) The computed tomography is showing a segment of the EKOS EndoWave catheter inside the iliac vein. 


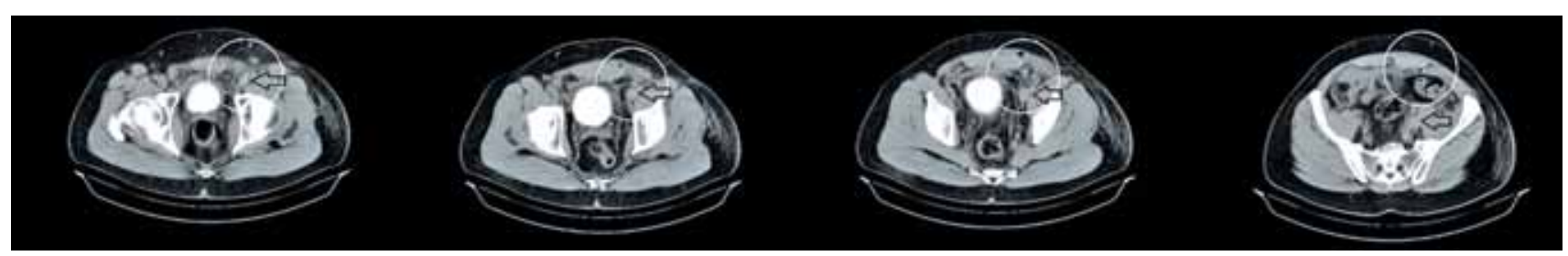

Figure 3. Consecutive slices of the computed tomography angiography showing the course of the EKOS EndoWave catheter during the extravasation from the iliac vein. Note the arrows are pointing at the iliac vein. The circles are showing the route the catheter tip took while passing out of the iliac vein into the abdominal wall.

bleed. The patient's total blood count was monitored for the detection of blood loss through the perforated iliac vein wall. Fortunately, no physical or hemodynamic changes implicating an active hemorrhage were observed. The patient was mobilized 12 hours later with no tenderness or hematoma on his abdominal wall.

Since the ultrasonic treatment was left undone, an intravenous (unfractionated heparin $15 \mathrm{IU} / \mathrm{kg} / \mathrm{h}$ ) and oral (calcium dobesilate and warfarin) medication was continued for 10 days until a regression of the symptoms was observed. After the recession of symptoms, the patient was discharged with a scheduled future follow-up. Oral warfarin $(5 \mathrm{mg} / \mathrm{day})$ was prescribed to keep the international normalized ratio (INR) between 2.0 to 2.5 .

\section{DISCUSSION}

Massive DVT and the $\mathrm{PE}$ are life-threatening conditions associated with a high incidence of fatalities comparable to that of acute myocardial infarction. Its clinical course can vary from a mild leg edema to a prolonged hospitalization with lifelong morbidity related to the PE and cardiopulmonary dysfunction or even mortality. ${ }^{[6]}$ The conventional treatment for acute DVT is immediate anticoagulation with lowmolecular-weight heparin or unfractionated heparin. It is then followed by an oral anticoagulation with warfarin for three to six months. ${ }^{[5]}$ This treatment aims to prevent thrombus propagation and to reduce the risks of PE and DVT recurrence. Although anticoagulation with unfractionated or low-molecularweight heparin followed by long-term warfarin therapy is widely regarded as the gold standard, this treatment does not provide a significant fibrinolytic activity in the patients with severe, extensive, proximal DVTs who have a high risk of developing subsequent $\mathrm{PE}$ or a PTS. ${ }^{[6]}$
Different treatment modalities have been used in patients with massive DVT or PE, including the systemic anticoagulation, surgical embolectomy, catheter directed thrombolysis, and percutaneous mechanical thrombectomy. ${ }^{[6]}$ Prior to the invention of catheter-based interventional techniques, anticoagulation was the only therapeutic modality used in massive DVT. However, anticoagulation has no direct thrombolytic effect. ${ }^{[7]}$ As a result, anticoagulation-based DVT treatments often do not restore the venous patency, causing permanent damage to the venous valves. ${ }^{[5]}$ In addition, eventual venous stenoses are predisposed to recurrent thrombosis and may cause May-Thurner syndrome if left untreated. ${ }^{[4]}$ The combination of venous obstruction and venous reflux significantly increases the risk of PTS. However, catheter-directed mechanical thrombolysis can remove the thrombus and restore the venous patency and may prevent a recurrent thrombosis, $\mathrm{PE}$ or PTS. ${ }^{[6]}$

The EKOS EndoWave Infusion Catheter System (EKOS Corporation, Bothell, WA) is a catheter directed thrombolysis in which the fibrinolytic process acceleration is done via ultrasound waves. The EndoWave System, produced by EKOS Corporation, consists of a $5.2 \mathrm{~F}, 106-\mathrm{cm}-$ long infusion catheter, an ultrasound treatment core catheter, and a control unit with the interface cables. A series of laser-cut microinfusion pores are located along the external infusion catheter for the delivery of the thrombolytic drug. The core catheter consists of microsonic transducer elements $(2 \mathrm{MHz}, 0.45 \mathrm{~W})$ that were distributed evenly $1.0 \mathrm{~cm}$ apart from each other along its leading tip. They deliver the ultrasound energy radially along the coaxial infusion zone. The microinfusion pores are positioned to optimize the interaction with the corresponding ultrasound transducers. This improves the efficiency of the thrombolytic process and it decreases the treatment time and the administered lytic dose. Moreover, the overall expense and the 
risk of associated complications such as bleeding are reduced. ${ }^{[8]}$

This extravasation of the catheter by perforating the iliac vein was the first such complication of the EKOS EndoWave system encountered thus far and no similar case was found in the recent literature. The probable mechanism is thought to be mechanical. A sudden flexor movement of the thigh might have conducted a direct force to the catheter. This axial force might have caused a forward displacement of the whole catheter. As a result, the bare tip of the catheter displaced and ripped the iliac vein and went outside through the abdominal muscles.

As a conclusion, EKOS EndoWave catheters should always be secured to avoid sudden movements of the implanted limb. Elevation of the leg or the body should be restricted to a particular angle that does not disturb the alignment of the catheter inside the vein. If movement of the patient becomes inevitable, the system should be temporarily shut down. If an iliac vein injury occurs during the catheter-based thrombolysis, the catheter should be withdrawn immediately, and the administration of anticoagulant or thrombolytic agents should be stopped as soon as possible. The patient's blood count should be monitored, and an abdominal examination should be repeated at short intervals during the first 24 hours.

\section{Declaration of conflicting interests}

The authors declared no conflicts of interest with respect to the authorship and/or publication of this article.

\section{Funding}

The authors received no financial support for the research and/or authorship of this article.

\section{REFERENCES}

1. Enden T, Haig Y, Kløw NE, Slagsvold CE, Sandvik L, Ghanima W, et al. Long-term outcome after additional catheter-directed thrombolysis versus standard treatment for acute iliofemoral deep vein thrombosis (the CaVenT study): a randomised controlled trial. Lancet 2012;379:31-8.

2. Garg K, Cayne N, Jacobowitz G. Mechanical and pharmacologic catheter-directed thrombolysis treatment of severe, symptomatic, bilateral deep vein thrombosis with congenital absence of the inferior vena cava. J Vasc Surg 2011;53:1707-10.

3. Köksoy C, Yilmaz MF, Başbuğ HS, Calik ES, Erkut B, Kaygin MA, et al. Pharmacomechanical thrombolysis of symptomatic acute and subacute deep vein thrombosis with a rotational thrombectomy device. J Vasc Interv Radiol 2014;25:1895-900.

4. Grommes J, Strijkers R, Greiner A, Mahnken AH, Wittens $\mathrm{CH}$. Safety and feasibility of ultrasound-accelerated catheter-directed thrombolysis in deep vein thrombosis. Eur J Vasc Endovasc Surg 2011;41:526-32.

5. Karthikesalingam A, Young EL, Hinchliffe RJ, Loftus IM, Thompson MM, Holt PJ. A systematic review of percutaneous mechanical thrombectomy in the treatment of deep venous thrombosis. Eur J Vasc Endovasc Surg 2011;41:554-65.

6. Lin $\mathrm{PH}$, Annambhotla S, Bechara CF, Athamneh $\mathrm{H}$, Weakley SM, Kobayashi K, et al. Comparison of percutaneous ultrasound-accelerated thrombolysis versus catheter-directed thrombolysis in patients with acute massive pulmonary embolism. Vascular 2009;17 Suppl 3:S137-47.

7. Öztürk C, Özalp B, İpeksoy Ü, Halıcı Ü. A new modality in the treatment of deep vein thrombosis: catheter-directed ultrasound-accelerated thrombolysis. Turk Gogus Kalp Dama 2014;22:755-60.

8. Owens CA. Ultrasound-Enhanced Thrombolysis: EKOS EndoWave Infusion Catheter System. Semin Intervent Radiol 2008;25:37-41. 